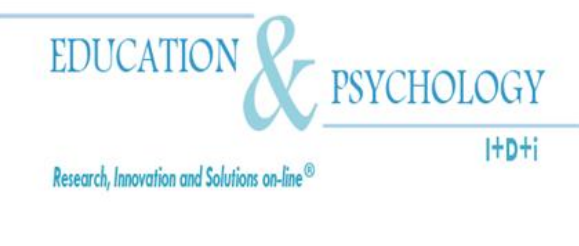

\title{
Psicología y Orientación Vocacional en España. Estudio Cientimétrico
}

\section{Raquel Flores Buils, José Manuel Gil Beltrán, Antonio Caballer Miedes, Miguel Ángel Martínez Martínez}

Departamento de Psicología Evolutiva, Educativa, Social y Metodología. Universitat Jaume I, Castellón de la Plana

\section{España}

Correspondencia: Raquel Flores Buils. Dep. Psicología Evolutiva, Educativa, Social y Metodología. Universitat Jaume I. Avd. Vicent Sos Baynat, s/n, CP. 12071, Castellón de la Plana.

E-mail: flores@psi.uji.es

(C) Education \& Psychology I+D+i and Editorial EOS (Spain) 


\section{Resumen}

Introducción. El estudio de la actividad investigadora es posible recurriendo a la cuantificación de determinadas variables presentes en los artículos publicados en las revistas científicas. Una vez cuantificadas obtenemos cifras que reflejan resumidamente las características de la actividad investigadora. Estas cifras las obtenemos a través de los Indicadores Cientimétricos. Se trata de un método objetivo y verificable, cuyos resultados son reproducibles, y que puede aplicarse a un gran volumen de datos, lo que hace posible la obtención de resultados significativos en los estudios estadísticos.

Método. En el presente trabajo llevamos a cabo un estudio cientimétrico de la Psicología y Orientación Vocacional en España (1990-2008), a través del análisis de revistas científicas: Revista Española de Orientación y Psicopedagogía, Bordón. Revista de Pedagogía, Revista de Investigación Educativa y Revista de Psicología General y Aplicada. De estos artículos, hemos seleccionado aquellos que hacen referencia expresa a la Orientación Vocacional, con el fin de realizar un análisis cientimétrico de los mismos. Así nos hemos centrado, por un lado, en el estudio de la Productividad Personal, Productividad Institucional, en el Análisis del Contenido y de los Instrumentos que los investigadores han utilizado en sus trabajos, y por otro lado, en el estudio de las Referencias Bibliográficas.

Resultados. De los resultados obtenidos, señalar que la mayor parte de los autores que publican en España, dentro de este ámbito, pertenecen al ámbito universitario nacional. Las temáticas más relevantes han sido la de Asesoramiento Vocacional y Orientación Vocacional, y, Programas/Sistemas de Intervención.

Discusón y conclusiones. Este tipo de estudios facilitan la comunicación directa entre los investigadores y los profesionales de la Orientación, permitiendo que la ciencia llegue a todos.

Palabras Clave: Psicología Vocacional, Orientación Vocacional en España, Estudios cientimétricos, TIC. 


\title{
Vocational Guidance and Psychology in Spain: Scientometric Study
}

\begin{abstract}
Introduction. Studies into research activity are possible by quantifying certain variables present in articles published in specialist journals. Once quantified, numerical data are obtained which summarise research activity characteristics. These data are obtained through scientometric indicators. This is an objective and verifiable method whose results can be both reproduced and applied to a large amount of data, thus allowing significant results to be obtained in statistical Studies.
\end{abstract}

Method. In this work we carried out a scientometric study on Psychology and Vocational Guidance in Spain (1990-2008) by analysing the following scientific journals: Revista Española de Orientación y Psicopedagogía (REOP), Bordón. Revista de Pedagogía, Revista de Investigación Educativa (RIE) and Revista de Psicología General y Aplicada. Of these articles, we selected those that expressly refer to vocational guidance, and we centred the study on personal productivity, institutional productivity, and on analysing the contents, instruments that researchers used in their works and references.

Results. From these results, we note that most of the authors who publish in Spain in this area belong to the national university. Also, the most relevant issues has been to Vocational counselling / vocational guidance, and Program / Systems Intervention.

Discussion and conclusion. Also, we stress that these studies provide direct communication between researchers and guidance professionals, allowing science reaches everyone.

Keywords: Vocational Psychology, Vocational guidance in Spain, Scientometric indicators, ICT.

Received: 10/30/12

Initial acceptance: 01/21/13

Final acceptance: 03/10/13 


\section{Introducción}

Dentro del conjunto de influencias que la ciencia de la ciencia ha recibido es importante destacar a la "Ciencia de la Documentación", cuyos orígenes fueron el intento de control de la ingente cantidad de documentación que se empezaba a producir en las diferentes áreas del saber, allá a finales del siglo XIX, y cuyos principales responsables de su nacimiento fueron los belgas P. Otiet y H. Lafontaine. En los años sesenta, y a partir de la teoría de la recuperación de información de Vickery (1948), la ciencia de la Documentación se fue transformando en una ciencia informativa (Information Science), fundamentalmente por la influencia anglosajona, pero también alemana y soviética. La aparición de esta disciplina tuvo una gran importancia para el desarrollo de la bibliometría y de la cienciometría, herramientas metodológicas del programa de la ciencia de la ciencia (Terrada, 1983). Esta influencia ha sido mayor a partir del desarrollo, en los últimos años, de las nuevas tecnologías de la información que han permitido la creación de grandes bases de datos científicas. Especial mención merece la fundación del ISI (Institute for Science Information, Filadelfia) por Garfíeld y la publicación de los conocidos índices de referencias (Science Citation índex, Social Science Citation index).

La Cienciometría, "Scientometrics", puede ser tratada también como un concepto análogo al de Bibliometría. Es una nueva área emergente de investigación. Utiliza técnicas de medición para evaluar el progreso de la Ciencia y su nivel de desarrollo, su impacto y relevancia en la sociedad. Es originaria de la antigua Unión Soviética y su primer desarrollo lo ha conocido en la Europa del Este. Involucra tanto a investigadores procedentes de diversos campos científicos como a responsables de organismos gubernamentales. El término fue definido por Dobrov (1966) como "medición de los procesos informáticos", entendiendo la informática en su acepción eslava es decir "estudio de la estructura y propiedades de la información científica y las leyes de los procesos de la comunicación científica" (citados por Sengupta, 1992). Es un campo interdisciplinar no en el sentido de que se ocupe de un tema más o menos especifico ubicado entre los límites de dos disciplinas tradicionales, sino en el sentido de que involucra a un gran número de disciplinas dada la amplitud de sus objetivos. Entre los especialistas que han intentado concretar una definición de la especialidad podemos citar a Vinckler: "la Cienciometría es una disciplina científica dedicada a los aspectos cuantitativos de la Ciencia y la investigación científica" (Vinkler, 1991). 
Dobrov afirmaba que la mayor atención de la Cienciometría se ha concentrado en el análisis de los parámetros "informacionales" del desarrollo científico, tales como número de trabajos, revistas, autores, por un lado y las leyes: envejecimiento, dispersión, estructura, flujos de documentos, procesos de la citación, etc., por otra. Desde este punto de vista el objetivo sería: “determinar la serie de índices dinámicos que describen el sistema de la ciencia en el proceso de su desarrollo teniendo en cuenta que la Ciencia es un sistema de probabilidad y sus resultados tienen una naturaleza probabilística" añade el mismo autor.

Así mismo, desde una perspectiva institucional, la revista es el lugar donde el trabajo científico tiene posibilidades de ser publicado, adquirir una existencia social y ser conservado. De este modo, las revistas expresan el estado de la ciencia en un momento determinado, revelan los temas que preocupan, los autores o grupos más activos y amplios, y las obras de mayor influencia. En definitiva, un conjunto de datos imprescindibles para conocer la situación de una disciplina.

La llegada de los sistemas automatizados en la década de los sesenta transformó los sistemas de almacenamiento de la información. Este proceso de almacenamiento y recuperación de la información ha estado estrechamente ligado al desarrollo de las tecnologías, siendo actualmente cuando se ha producido el cambio más significativo y el que ha transformado completamente la industria de la información.

Así, si la conceptualización de la Ciencia está evolucionando de manera rápida, también la Orientación Vocacional a pesar de su juventud (los autores están de acuerdo en que se inicia con Parson en 1909 tras la publicación de su obra Choosing a vocation) está sufriendo profundos cambios para adaptarse a la nueva realidad social. De ahí nuestra preocupación por dar respuesta a una serie de cuestiones que siempre nos ha llamado la atención: ¿Qué pasa con la Orientación Vocacional? ¿Se habla mucho y se hace poco? ¿La sociedad, los legisladores, etc., le dan gran importancia y luego no se desarrolla? ¿Realmente dónde estamos? ¿Hacia dónde deberíamos dirigir la intervención en el proceso de asesoramiento para ser eficaces?

En los últimos años la Psicología Vocacional no sólo ha ido acumulando literatura científica de interés, sino que también ha ido dando ciertos pasos profesionales y políticos. Pese a esto, todavía posee algunas deficiencias o lagunas, (por ejemplo, que existen ciertos temas y poblaciones que han sido poco estudiados, que los vínculos con otros ámbitos de in- 
vestigación a menudo se han perdido, o que no se ha prestado mucha atención a las variables contextuales y culturales). Una serie de amenazas y oportunidades aguardan a la psicología vocacional en la próxima década. Algunas de las amenazas (por ejemplo, la disponibilidad de servicios basados en Internet) también representan grandes oportunidades, en función de cómo se planifiquen. Dentro de este contexto.

\section{Objetivos}

Con el trabajo que hemos realizado podemos de manifiesto las fortalezas y debilidades de la Psicología y Orientación Vocacional en nuestro país respecto a las necesidades de la sociedad actual. De ahí que nos planteemos dos grandes objetivos en nuestra investigación. El primero, conocer la producción científica en España del área de Orientación Vocacional en el periodo comprendido entre 1990-2008, dando con ello continuidad a los trabajos de:

- 25 años de investigaciones españolas sobre Orientación Vocacional. Revisión bibliográfica sobre Orientación Vocacional (1952-1978), de Carlos Castaño López-Mesas.

- Psicología Escolar, Psicología Vocacional y Orientación Escolar a través de las Revistas Bordón, Revista Española de Pedagogía y Revista de Psicología General y Aplicada. (1970-1985), de Rafael Reig Peiró.

- Orientación y Psicología Vocacional en España: Revisión y estado de la cuestión (1970-1999), de $\mathrm{M}^{\mathrm{a}}$ Teresa Adame Obrador.

El análisis de estos estudios previos nos aporta una gran fuente de información sobre las temáticas, materiales, recursos, métodos de investigación, etc., que se han utilizado en el área de Psicología Vocacional a través de estos años en España.

El segundo objetivo, desarrollar una Base de Datos de Investigación en Orientación Vocacional (Flores, Gil, Caballer y Martínez, 2012) que no tuviese como único objetivo el registro de información, sino que su finalidad fuese la de constituirse en una herramienta para la investigación en base a los indicadores cientimétricos. 


\section{Método}

\section{Muestra}

La investigación se basa en los datos aportados tras el estudio de las siguientes Revistas de Investigación:

a) Revista Española de Orientación y Psicopedagogía (REOP): órgano de expresión de la Federación Española de Orientación y Psicopedagogía (FEOP)

b) Bordón. Revista de Pedagogía: publicada por la Sociedad Española de Pedagogía (SEP)

c) Revista de Investigación Educativa (RIE): perteneciente a la Asociación Interuniversitaria de Investigación Pedagógica (AIDIPE).

d) Revista de Psicología General y Aplicada: primera revista publicada en España dirigida al campo de la Psicología y que fue fundada por el Dr. Germain en el año 1927.

Las cuatro Revistas españolas han sido seleccionadas en base al criterio cientimétrico de gemelización. Son Revistas que tienen ciertas características comunes que permiten realizar una serie de estudios comparativos. Es bien cierto que la Revista de Psicología General y Aplicada no encaja con todos los aspectos de la gemelización, ahora bien, su selección se ha hecho siguiendo el criterio de que fue la representante de la primera Sociedad Española de Psicología (SEP), convirtiéndose así en un exponente científico de la Sociedad. Es por ello que la Revista posee un número elevado de artículos que no se centran en Orientación Vocacional, ya que abarca todas las áreas de la Psicología.

De ellas, hemos analizado todos los artículos publicados durante el mismo periodo, de 1990 a 2008. Así tenemos: Revista Española de Orientación y Psicopedagogía, 331; Revista de Investigación Educativa (RIE), 558; Bordón. Revista de Pedagogía, 664 y la Revista de Psicología General y Aplicada, 305. Los artículos fueron clasificados a partir de un sistema de jueces teniendo en cuenta la conceptualización teórica (Flores, 2010) en los ámbitos de Orientación Educativa, Orientación Vocacional, Orientación Profesional, Salud y Metodología.

Para la presente investigación, de la muestra expuesta, seleccionamos aquellos artículos comprendidos en el área de Orientación Vocacional. Para ello, hemos partido de los supuestos teóricos propuestos por Castaño (1983) y Rivas (2003) respecto a la Orientación Vo- 
cacional y Psicología Vocacional respectivamente. De esta manera, la muestra definitiva está formada por (ver Tabla 1):

Tabla 1. Muestra del estudio

\begin{tabular}{|c|c|c|c|c|c|}
\hline Revista & $\begin{array}{l}\mathrm{N}^{\circ} \text { Artí- } \\
\text { culos }\end{array}$ & $\begin{array}{l}\mathrm{N}^{\circ} \mathrm{Au}- \\
\text { tores }\end{array}$ & $\begin{array}{c}\mathrm{N}^{\circ} \\
\text { Firmas }\end{array}$ & $\begin{array}{l}\mathrm{N}^{\circ} \text { Institucio- } \\
\text { nes }\end{array}$ & $\begin{array}{l}\mathrm{N}^{\circ} \text { Referencias Bi- } \\
\quad \text { bliográficas }\end{array}$ \\
\hline $\begin{array}{l}\text { Revista Española de Orientación y } \\
\text { Psicopoedagogía (REOP) }\end{array}$ & 54 & 78 & 89 & 27 & 1032 \\
\hline $\begin{array}{l}\text { Revista de Investigación Educativa } \\
\text { (RIE) }\end{array}$ & 40 & 61 & 84 & 16 & 1005 \\
\hline Bordón. Revista de Pedagogía & 18 & 27 & 34 & 7 & 378 \\
\hline $\begin{array}{l}\text { Revista de Psicología General y } \\
\text { Aplicada }\end{array}$ & 8 & 13 & 17 & 6 & 167 \\
\hline Total & 120 & 179 & 224 & 56 & 2582 \\
\hline
\end{tabular}

\section{Procedimiento: Indicadores Cientimétricos}

Los indicadores que se han utilizado en la presente investigación son:

1. Productividad personal: se analiza el número de trabajos publicados por cada autor a lo largo del periodo de tiempo estudiado.

2. Productividad institucional: hace referencia al número de trabajos publicados según la institución de trabajo de los autores a lo largo del periodo estudiado.

3. Estudio de contenido: éste se adscribe a las distintas materias y representan el mejor indicador de la orientación científica de las Revistas, ya que ponen de manifiesto las temáticas que éstas cubren.

Para el estudio de contenido, diseñamos una ficha que recoge una serie de variables que nos permiten analizar todos los aspectos que intervienen en este indicador (Flores, 2007). Dichas variables son:

a) Área: Orientación Vocacional

b) Campo/Temática: Definimos los campos que han tenido mayor incidencia en la producción científica, para ello, utilizamos la "Clasificación Temática de Orientación Vocacional" (Flores, 2010), que es una actualización de la propuesta de Adame (2000). 
c) Incidencia: Nos indica el ámbito donde el artículo tiene su mayor grado de aplicación: adolescencia, orientadores, profesores, adultos, familia, Bachillerato, Secundaria y Universidad.

d) Tipo: Clasificación de los artículos según si son teóricos, empíricos o prácticos. En los artículos empíricos, analizamos el diseño metodológico seguido.

e) Palabras clave: Análisis cualitativo de las palabras clave que aparecen en los artículos, determinando aquellas que han sido más utilizadas.

4. Referencias Bibliográficas: nos permite conocer cuáles han sido, tanto los documentos como los autores más referenciados en la bibliografía utilizada en los artículos objeto de análisis. Ello implica conocer las tendencias teóricas y los autores más influyentes en el campo de la Orientación Vocacional.

Finalmente, pese a que no es en sí mismo un indicador cientimétrico, hemos analizado los Instrumentos que han sido utilizados por los autores de los artículos en sus estudios, clasificándolos en: pruebas, instrumentos de elaboración propia, programas y material TIC.

\section{Instrumento}

Para el análisis de estos indicadores hemos desarrollado y utilizado la Base de Datos de Investigación para la Orientación Vocacional (Flores, 2010; Flores, Gil, Caballer y Martínez, 2012), la cual no sólo registra todos los artículos que forman parte de nuestra muestra sino que también nos facilita la realización de estudios cientimétricos.

Posee un Módulo de Administración y otro para los Usuarios. Al Módulo de Administración sólo tienen acceso los miembros del equipo de investigación. Entre las diferentes funciones que podemos realizar, encontramos:

1) Mantenimiento de las tablas maestras: A través de este apartado tenemos acceso a los datos introducidos en la Base de Datos. De esta manera podemos tanto introducir datos nuevos como modificar los ya existentes. Estas tablas maestras son dinámicas ya que permiten la actualización inmediata de los datos ante cualquier modificación de los mismos, de manera que facilitan el cálculo automático de las distintas variables que integran esta base de datos. 
2) Mantenimiento de los artículos: Desde aquí se puede modificar, añadir o eliminar datos. Estos datos se verán reflejados directamente en las tablas maestras. Cada artículo incluye la siguiente información: Revista a la que pertenece, páginas, volumen, número y año; Título artículo; Autores; Resumen; Área a la que pertenece; Tipo de artículo (estudio o experiencia); Metodología que sigue (teórico, empírico o práctico); Idioma; Si posee anexos de interés; Campo al que pertenece; Incidencia y Palabras clave. Desde este apartado podemos realizar búsquedas generales por uno o varios campos de los que acabamos de ver.

3) Consultas e informes: Podemos realizar búsquedas tanto por Palabras Clave como por autores. En ambos casos, se facilitan los Glosarios con los datos. Es importante señalar la aportación de un Glosario de Palabras Clave realizado por nosotros con términos relacionados con la Orientación Vocacional. Éste comprende 245 términos que se presentan en tres idiomas (español-inglés-francés), de esta manera se facilita la búsqueda de artículos en cualquiera de estos tres idiomas evitando errores de traducción de una lengua a otra.

4) Resultados: Este apartado nos permite cuantificar los datos de las distintas variables que conforman la base de datos (datos y gráficas).

Dentro del Módulo de Usuario, se permite realizar los tres tipos de consultas que hemos visto en anteriormente. También se tiene acceso a los Glosarios.

\section{Análisis de datos}

Hemos realizado una investigación sistemática, controlada, empírica y crítica sobre el estado actual de la Orientación Vocacional a través de los artículos publicados en diversas revistas de investigación. Para ello hemos utilizado un tipo de trabajo empírico con un diseño descriptivo, atendiendo a la descripción de la situación del área de Orientación Vocacional

\section{Resultados}

\section{Análisis de la Productividad Personal}

Si analizamos la relación entre el número de artículos publicados y el número de firmantes, se observa como en cinco de los diecinueve años analizados el número de autores respecto al número de artículos publicados es superior al doble, en siete de ellos el número de firmantes es prácticamente el doble y sólo durante cinco años la diferencia es mínima. De estos datos se desprende la tendencia de los autores españoles a realizar sus investigaciones en equipo. De este análisis global pasamos a identificar a cada autor de manera individual. Para 
ello, presentamos la Tabla 2, con los autores que más trabajos han publicado en total en las cuatro Revistas analizadas y su índice de productividad. El Índice de Productividad lo hemos calculado siguiendo a Lotka: $I P=\log$ de la productividad.

Tabla 2. Productividad de los autores de las Revistas analizadas

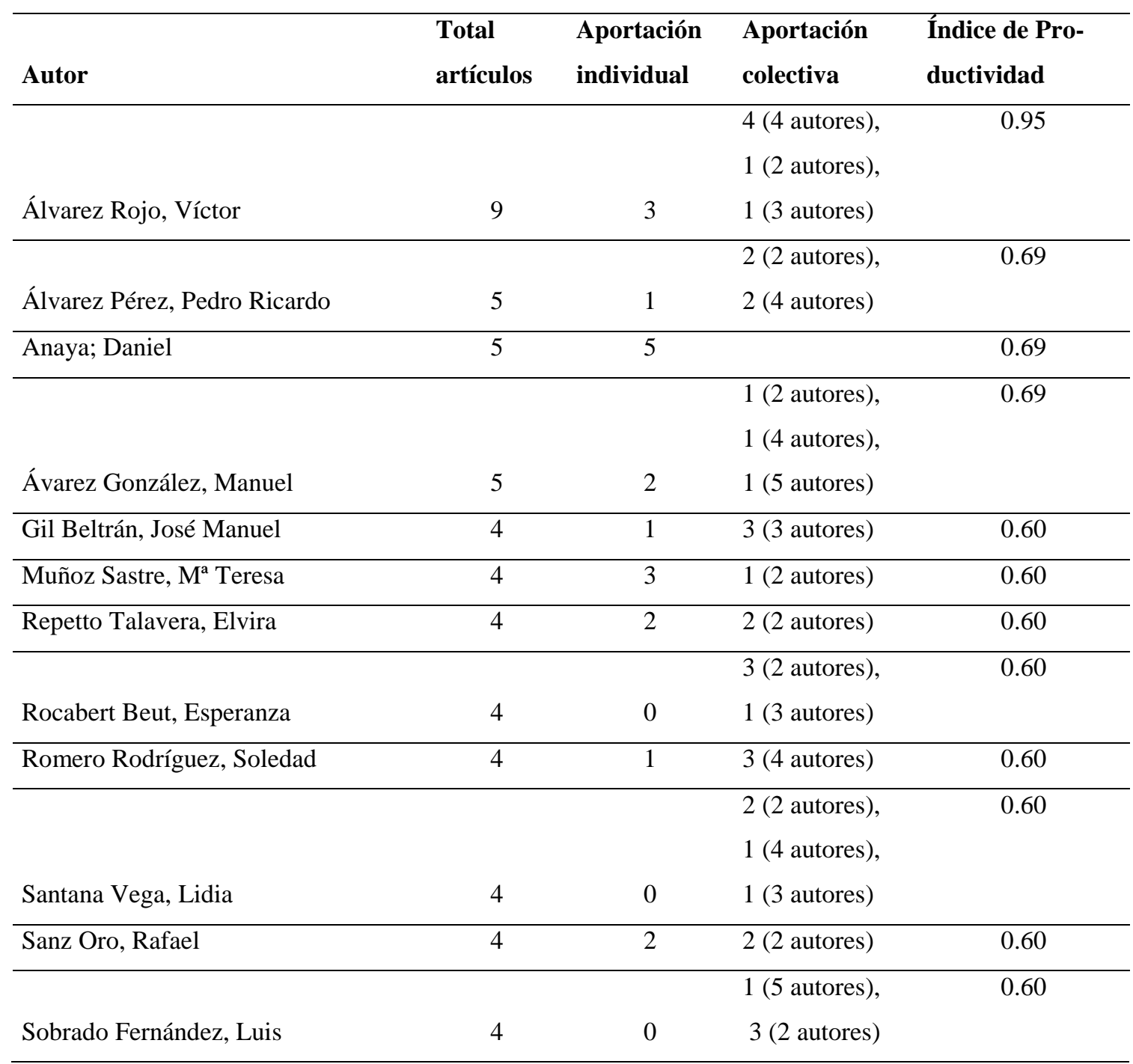

Respecto a los autores, observamos que el autor más productivo es Álvarez Rojo, con una producción total de 9 artículos de los cuales tres los firma individualmente y los restantes son trabajos colectivos en los cuales han intervenido dos, tres o cuatro autores. Un dato a destacar es que todos estos autores que han publicado sus trabajos en las Revistas analizadas, son todos españoles, no aparece ningún autor extranjero. Esto refleja un carácter eminentemente centrado en España, no recogiendo de manera significativa trabajos de investigación que se estén realizando fuera de nuestro país. 
De estos autores, hemos llevado a cabo la estimación de la producción bibliográfica de una persona. Cuando estos forman parte de una autoría múltiple la hemos llevado a cabo a través de la cuenta completa (Pascual, 1981). Así encontramos que del total de 120 artículos publicados sobre Orientación Vocacional en las cuatro Revistas durante el periodo analizado, la productividad se centra fundamentalmente en los artículos con una firma (28.5\%), dos firmas $(27.6 \%)$, tres firmas $(16.74 \%)$, cuatro firmas $(15.83 \%)$, cinco firmas $(9.04 \%)$ y seis fir$\operatorname{mas}(2.26 \%)$.

Un aspecto fundamental en este estudio de la multiautoría es el índice de colaboración (IC). Este es el indicador más utilizado para referirse al grado de co-autoría entre colegas, puesto que se trata de una única cifra que indica qué número de firmas por término medio ha intervenido en los artículos o trabajos. Para ello se determina la razón entre el número de firmas y el número de artículos. Si nos fijamos en el I.C. se deduce como este ha ido aumentando de manera irregular (años alternativos de alto y bajo) a lo largo del tiempo. Sin embargo aparece un claro aumento en los periodos de 1990 a 1993 y entre 1999 al 2002, apareciendo después de este último año una tendencia a bajar.

Ahora bien, la media de este índice es de 1.8, no llegando a 2, siendo éste el valor promedio dentro de las Ciencias de la Educación (Fernández y Bueno, 2002). Sin embargo, podemos apreciar que durante ocho años el I.C. es igual o superior a esta cifra, destacando el año 2002 con un I.C. de 3.2. En definitiva, los valores que hemos hallado, son casi una constante en la productividad de las Ciencias de la Educación.

\section{Análisis de la Productividad Institucional}

En este apartado nos centraremos en las Instituciones que han aportado un mayor número de artículos al conjunto de las Revistas españolas analizadas. Así, las universidades que más artículos han aportado, son: Sevilla (15), UNED (Universidad Nacional de Estudios a Distancia) (12) y la Universidad de Barcelona (10).

De las 29 Instituciones que han publicado sus trabajos sobre Orientación Vocacional en estas Revistas, hay que destacar que todas son universidades. Así mismo, la mayor parte de ellas son españolas, sólo 6 pertenecen a otros países. 


\section{Análisis de Contenido}

\section{Cатро}

Teniendo en cuenta la Clasificación Temática de Orientación Vocacional (Flores, 2010), el campo más representado es el de Programas/sistemas de intervención y asesoramiento/orientación de la conducta vocacional, con 33 aportaciones. A este le siguen el de Asesor, equipos y servicios de orientación vocacional (13), Origen y desarrollo histórico (12), Conducta vocacional/vocación: concepto, teorías vocacionales (9), y Elección vocacional (8).

\section{Incidencia}

Si nos centramos en la incidencia que han tenido los trabajos publicados, podemos observar como éstos están dirigidos a: estudiantes de Secundaria (31), Orientadores (30) y Universidad (22). Siguiendo con el análisis de la incidencia de los artículos, pero desglosada según el campo al que pertenece cada trabajo, encontramos los siguientes datos reflejados en la Tabla 3.

Tabla 3. Campos objeto de estudio según la incidencia en las Revistas analizadas

\begin{tabular}{|c|c|c|c|c|c|c|c|c|}
\hline Campo / Incidencia & $\begin{array}{l}\frac{\pi}{0} \\
\frac{0}{0} \\
\frac{0}{0} \\
\frac{0}{0} \\
\frac{0}{\pi}\end{array}$ & $\frac{\stackrel{\mathscr{O}}{Z}}{\frac{Z}{\mathbb{Z}}}$ & 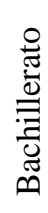 & 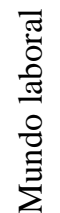 & 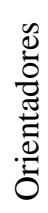 & 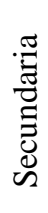 & $\stackrel{0}{0}$ & $\begin{array}{l}7 \\
0 \\
0 \\
0 \\
0 \\
0\end{array}$ \\
\hline
\end{tabular}

A.2. Conducta vocacional / vocación: concepto, teorías vocacionales

A.3. Asesoramiento vocacional / orientación vocacional

11

$\begin{array}{llllll}5 & 2 & 1 & 1 & & 9 \\ & & & 1 & & 3\end{array}$

A.3.1. Enfoques del asesoramiento / orientación vocacional: fundamentos teóricos-tecnológicos

A.3.2. Diagnóstico / evaluación vocacional

A.3.3. Programas / sistemas de intervención y asesoramiento / orientación de la conducta vocacional

A.3.4. Asesor, equipos y servicios de orientación vocacional

$\begin{array}{lllllll}3 & 1 & 2 & 5 & 15 & 7 & 33\end{array}$

A.3.5. Asesoramiento / orientación vocacional en el curriculum escolar

2

A.4. Desarrollo vocacional / desarrollo de la carrera

A.4.10.2. Minorías funcionales: deficiencias físicas, psíquicas y sensoriales

A.4.3. Intereses / preferencias vocacionales

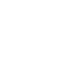


A.4.4. Toma de decisiones vocacionales

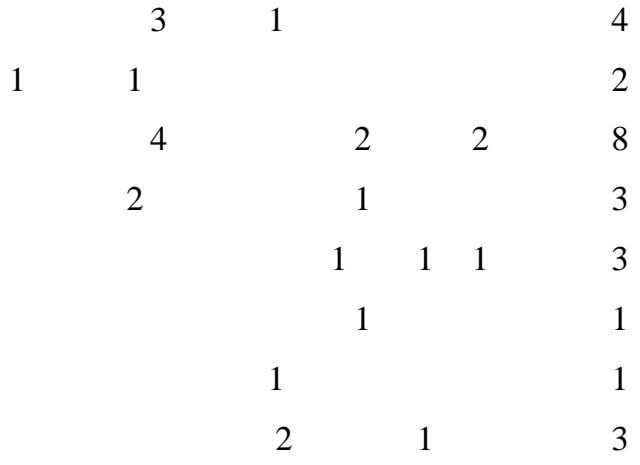

A.7. Asesoramiento / orientación vocacional por países,

comunidades / situación comparativa. $\quad 1 \quad 1 \quad 2$

\begin{tabular}{llllllllll}
\hline Total & 1 & 2 & 11 & 1 & 8 & 14 & 7 & 10 & 120
\end{tabular}

Como se desprende de los datos, destaca el campo de Programas/sistemas de intervención y asesoramiento/orientación de la conducta vocacional dirigido a Secundaria y en segundo lugar hay que destacar el campo de Origen y desarrollo histórico dirigido a los Orientadores.

\section{Tipo}

Respecto al tipo de artículo, en general, aparece un predominio de los artículos de corte Empírico seguidos de los Teóricos. Así mismo los artículos Prácticos representan un número muy bajo respecto al resto. Tras el estudio del tipo de diseño que se ha utilizado en los trabajos empíricos, presentamos los resultados en la Tabla 4.

\section{Tabla 4. Tipo de estudios empíricos en las Revistas analizadas}

\begin{tabular}{lll}
\hline \multicolumn{1}{c}{ Tipo de estudio empírico } & $\mathrm{N}^{\mathbf{0}}$ & $\%$ \\
\hline Encuesta Transversal & 39 & $78 \%$ \\
Diseños Cuasiexperimentales & 3 & $6 \%$ \\
Diseños ex post facto & 2 & $4 \%$ \\
Entrevistas en Profundidad & 2 & $4 \%$ \\
Estudios Bibliométricos & 2 & $4 \%$ \\
Estudio Experimental de Comparación de Grupos & 1 & $2 \%$ \\
Diseño de Encuesta Longitudinal & 1 & $2 \%$ \\
\cline { 1 - 2 } Total & &
\end{tabular}


Observamos como todos los tipos de diseños utilizados en los artículos empíricos corresponden a metodologías experimentales y cuasiexperimentales y metodología de encuestas, no apareciendo ningún trabajo que siga una metodología observacional, ni cualitativa.

\section{Palabras Clave}

Respecto a las palabras clave encontramos que los autores de los artículos han utilizado un total de 353. Si llevamos a cabo un análisis cualitativo de las mismas, se aprecia que las palabras clave más utilizadas corresponden a: Orientación vocacional, Toma de decisiones, Educación Secundaria, Programas de Orientación, Servicio de Orientación, Orientación Universitaria, Universidad, Asesoramiento vocacional, Desarrollo de la carrera, Intereses vocacionales y Orientador.

\section{Análisis de las referencias Bibliográficas}

\section{Referencias por autor}

Si analizamos qué autores han recibido mayor número de referencias entre las Revistas analizadas que han formado parte de este estudio cientimétrico, nos encontramos con los datos que presentamos en la Tabla 5.

Tabla 5. Autores más referenciados en las Revistas analizadas

\begin{tabular}{lc}
\hline Autores más referenciados & $\mathrm{N}^{\circ}$ Referencias \\
\hline Repetto Talavera, E. & 43 \\
Super, D. E & 42 \\
Álvarez Rojo, V & 39 \\
Holland, J. L. & 37 \\
Rivas Martínez, F. & 34 \\
Rodríguez Moreno, M. L. & 33 \\
Rodríguez Espinar, S & 31 \\
Echevarria, B & 25 \\
Watts, A. G. & 24 \\
Sanz Oro, R. & 21 \\
Bisquerra, R & 20 \\
Álvarez, M & 19 \\
Gysberg, N.C. & 16 \\
Hoyt, K. B. & 16 \\
\hline
\end{tabular}


Como se puede observar, sólo cinco de ellos son autores extranjeros, siendo éstos considerados como los más representativos del área de la Psicología Vocacional. En cuanto a los autores españoles, se deduce que son los que mayor influencia ejercen tanto a nivel de fundamentación teórica para la investigación, como en la aplicación práctica por parte de los orientadores en su labor profesional.

\section{Tipo de Documento-Idioma}

El tipo de documento más utilizado para referenciar los artículos ha sido el Libro (1061), a continuación las Revistas de Investigación (955), y en tercer lugar encontramos los capítulos de libros (279). La mayor parte de estos documentos referenciados es el inglés (52.14\%), el español $(44.79 \%)$ y francés $(2.56 \%)$. El resto de idiomas es prácticamente testimonial. Un dato a señalar es que las Comunicaciones representan un alto índice de utilización en español. Éstas hacen referencia a las Actas de los Congresos y Jornadas celebrados en España. Aunque las cifras no sean elevadas, se aprecia una tendencia cada vez mayor a realizar referencias de documentos on-line. Esto es debido a que con la incorporación de las nuevas tecnologías, encontramos documentos y materiales que únicamente se insertan en la red.

\section{Año de Publicación del Documento de Referencia}

En este apartado analizamos las referencias utilizadas por los autores de los artículos de Orientación Vocacional, según el año de publicación del documento referenciado.

Respecto a la "Actualidad" de la bibliografía que se ha utilizado en los artículos publicados en el periodo analizado, observamos como las décadas más referenciadas son la que va del 1981 a 1990 y la comprendida entre 1991-2000. Si se observa estas referencias con el año de la Revista se comprueba que las referencias finales se centran en la bibliografía de los últimos diez años respecto al año de publicación del artículo, por lo que consideramos que la bibliografía utilizada es bastante actual.

\section{- Documentos de Referencia}

En este apartado realizamos un estudio sobre los documentos (libros y revistas de investigación) que han sido utilizados por los autores. Así, dentro de los libros españoles, el más referenciado es el Manual de Orientación y Tutoría de Álvarez González y Bisquerra con 14 referencias. Éste es considerado uno de los libros que más repercusión ha tenido entre los orientadores españoles. En segundo lugar encontramos el libro Calidad en la Universidad: 
Orientación y Evaluación de Apocada y Lobato, con 11 referencias, y en tercer lugar el libro Orientación e intervención psicopedagógica de Rodríguez Moreno con 10. El cuarto documento, corresponde a un libro en español Modelos de Orientación e Intervención Psicopedagógica de Bisquerra. Éste representa todo un clásico de la Orientación Vocacional en el ámbito de los orientadores españoles. Su utilización por parte de los autores ha sido a través de los Capítulos del Libro.

En cuanto a los libros extranjeros más referenciados, cabe destacar: Career Development Inventory de Super, D.E. y sus colaboradores; Comprehensive guidance programs that work de Gysberg, N.C. y Self-Directed Search de Holland, J. L.

Por otro lado, entre las Revistas españolas, encontramos: Revista Española de Orientación y Psicopedagogía con 52 referencias, Revista de Investigación Educativa (RIE) con 41, Bordón con 24 y Revista de Psicología General y Aplicada con 16; y entre las Revistas de otros países, están: la Journal of Vocational Behavior con 70 referencias, en segundo lugar aparece la School Counselor con 34 referencias, y en tercer lugar, con 29 referencias, la Journal of Counseling y Development.

\section{Discusión y conclusiones}

El ámbito de la Psicología y Orientación Vocacional, no sólo posee gran relevancia en el contexto educativo español, sino también en el ámbito internacional, ya que en la sociedad actual, especialmente en lo que hace referencia a la empleabilidad y movilidad de los estudiantes, tiene una gran implicación en los sistemas educativos de diferentes países. Así, diversos estudios como los que aparecen en la Tabla 6, ponen de manifiesto los tópicos de investigación que son más abordados por los investigadores. Tópicos que en definitiva, inciden directamente en un desarrollo de la carrera más eficaz de la persona, al tiempo que nos indican la dirección en la formación que los Orientadores deberían poseer para afrontar los retos que la sociedad del Siglo XXI les va a deparar. Así tenemos los estudios de (ver Tabla 6):

- Borgen (1991) analiza 1029 trabajos publicados entre 1971 y 1991 en la Journal of Vocational Behavior.

- Álvarez (1995) analiza 37 trabajos de la revista Career Development Quarterly y 102 de la Journal of Vocational Behavior durante el periodo 1987 a 1990. 
- Nilsson et al. (2007) analiza en su trabajo 990 artículos publicados en las siguientes revistas: Journal of Vocational Behavior (JVB, 1971-2004), Career Development Quarterly (CDQ, 1970-2004; anteriormente Vocational Guidance Quarterly); Journal of Career Assessment (JCA, 1993-2004); y Journal of Career Development (JCD, anteriormente Journal of Career Education, 1972, 1974-2004).

- Flores (2010) análisis de 561 artículos publicados en las revistas: Revista Española de Orientación y Psicopedagogía (1990-2008), Bordón. Revista de Pedagogía (1990-2008), Revista de Investigación Educativa (1990-2008), Revista de Psicología General y Aplicada (1990-2008), L'Orientation Scolaire et Professionnelle (1992-2008), Journal of Vocational Behavior (1990-2008) y la International Journal for Educational and Vocational Guidance (2001-2008).

Tabla 6. Temáticas más investigadas en Orientación Vocacional

\begin{tabular}{|c|c|c|c|}
\hline $\begin{array}{c}\text { Borgen } \\
(1991)\end{array}$ & Álvarez (1995) & Nilson, et al (2007) & Flores (2010) \\
\hline $\begin{array}{l}\text { Elección } \\
\text { ocupacional }\end{array}$ & $\begin{array}{l}\text { Madurez vocacio- } \\
\text { nal }\end{array}$ & $\begin{array}{l}\text { Países, poblaciones o culturas } \\
\text { especificas }\end{array}$ & $\begin{array}{l}\text { Programas / sistemas de } \\
\text { intervención y asesora- } \\
\text { miento / orientación de la } \\
\text { conducta vocacional }\end{array}$ \\
\hline $\begin{array}{l}\text { Satisfacción } \\
\text { laboral }\end{array}$ & Career Counseling & $\begin{array}{l}\text { Actitudes profesionales y } \\
\text { académicas }\end{array}$ & $\begin{array}{l}\text { Metodología de investiga- } \\
\text { ción }\end{array}$ \\
\hline $\begin{array}{l}\text { Desarrollo } \\
\text { vocacional }\end{array}$ & $\begin{array}{l}\text { Desarrollo de la } \\
\text { carrera en las or- } \\
\text { ganizaciones }\end{array}$ & $\begin{array}{l}\text { Intereses/aspiraciones/elección } \\
\text { de la carrera }\end{array}$ & $\begin{array}{l}\text { Conducta vocacional } \\
\text { vocación: concepto, teorías } \\
\text { vocacionales }\end{array}$ \\
\hline $\begin{array}{l}\text { Toma de } \\
\text { decisiones }\end{array}$ & $\begin{array}{l}\text { Orientación voca- } \\
\text { cional de las mi- } \\
\text { norías }\end{array}$ & Variables de personalidad & $\begin{array}{l}\text { Intereses / preferencias } \\
\text { vocacionales }\end{array}$ \\
\hline $\begin{array}{l}\text { Madurez } \\
\text { vocacional }\end{array}$ & $\begin{array}{l}\text { Toma de decisio- } \\
\text { nes }\end{array}$ & Desarrollo de la carrera & $\begin{array}{l}\text { Origen y desarrollo histó- } \\
\text { rico }\end{array}$ \\
\hline $\begin{array}{l}\text { Congruencia } \\
\text { indicadores }\end{array}$ & $\begin{array}{l}\text { Diagnóstico voca- } \\
\text { cional }\end{array}$ & $\begin{array}{l}\text { Asesoramiento/cliente/asesor de } \\
\text { la carrera }\end{array}$ & Instrumentación \\
\hline Estrés laboral & $\begin{array}{l}\text { Orientación voca- } \\
\text { cional asistida por } \\
\text { ordenador }\end{array}$ & Teorías/modelos & $\begin{array}{l}\text { Toma de decisiones voca- } \\
\text { cionales }\end{array}$ \\
\hline \multirow[t]{3}{*}{ Autoeficacia } & $\begin{array}{l}\text { Intereses vocacio- } \\
\text { nales }\end{array}$ & Estructura y tipo de carrera & $\begin{array}{l}\text { Asesoramiento / orienta- } \\
\text { ción vocacional por países, } \\
\text { comunidades / situación } \\
\text { comparativa }\end{array}$ \\
\hline & Valores de trabajo & $\begin{array}{l}\text { Toma de decisiones de la carrera } \\
\text { e indecisión }\end{array}$ & $\begin{array}{l}\text { Desarrollo vocacional } \\
\text { desarrollo de la carrera }\end{array}$ \\
\hline & Transición & Género y rol del sexo & $\begin{array}{l}\text { Enfoques del asesoramien- } \\
\text { to / orientación vocacional: } \\
\text { fundamentos teóricos- } \\
\text { tecnológicos }\end{array}$ \\
\hline
\end{tabular}


Del análisis de esta Tabla, se deduce que los tópicos de investigación más utilizados son: Toma de Decisiones, Intereses Vocacionales, Mundo Laboral, Desarrollo Vocacional y Asesoramiento Vocacional.

La investigación que hemos llevado a cabo sobre la Psicología y Orientación Vocacional en nuestro país, nos ha permitido constatar la potencia de la metodología cientimétrica para conocer la evolución y el estado de una ciencia; ya que esto es una de las maneras más importantes para avanzar no sólo en el conocimiento científico sino también su trasmisión a la Comunidad Científica. Así mismo creemos que otra aportación importante de esta investigación ha sido el desarrollo de la Base de Datos en Investigación en Orientación Vocacional, ya que se ha mostrado muy eficaz no sólo en el registro de la información, sino en el tratamiento de los datos, ya que permite la realización de informes y actualización inmediata de éstos. Así mismo permite realizar este tipo de análisis a otras áreas de la Orientación (educativa, profesional, etc.), así como a otras áreas de la Psicología (social, clínica, etc.).Teniendo en cuenta estas dos premisas generales queremos destacar de nuestro estudio los siguientes aspectos:

a) Las Revistas tienen un marcado carácter pedagógico tal como se pone de manifiesto al analizar la distribución de los artículos por áreas, ya que es el área de Orientación Educativa la más representativa. Al respecto, queremos constatar que no existe en nuestro país ninguna revista que dentro del campo de la Psicología sea representativa del área de Orientación Vocacional. Hecho que sí que ocurre con revistas de otros países.

b) La escasez de aportaciones de autores de otros países, pone de manifiesto el carácter eminentemente nacional de las investigaciones llevadas a cabo en nuestro país.

c) La mayor productividad se centra en dos periodos, años 1990 y 1992, y años 1998, 2001 y 2005. Esto creemos que puede ser debido a la aparición de dos leyes educativas en nuestro país como es la LOGSE (1990) en la que se da gran importancia a la Orientación, y por otra parte, la aparición de la LOE (2006) donde se sigue desarrollando la importancia de la Orientación y de los Servicios de Orientación; al mismo tiempo que la implantación de los Departamentos de Orientación en los centros de secundaria, es ya una realidad. Hay que señalar que hay una tendencia clara entre los autores españoles a escribir sus artículos en equipo; tal como se pone de manifiesto al obtener el I.C. de la productividad personal. 
d) En cuanto a las Instituciones que mayor productividad tienen son la Universidad de Sevilla, la Universidad Nacional de Educación a Distancia (UNED) y la Universitat de Barcelona.

e) Hay poca presencia de autores no universitarios, siendo la mayoría profesorado universitario. Esto consideramos que es un hándicap, ya que hay muy poca relación entre la práctica y la investigación. El investigador debe conocer la realidad y, el orientador debe conocer la investigación. Esto es uno de los aspectos que señala Savickas (1995) al proponer que uno de los aspectos de futuro de la Psicología Vocacional pasa por compartir la experiencia con la investigación; de ese modo las publicaciones podrían llegar a todos y no quedarse reducidas a una serie de revistas que sólo pueden ser leídas y consultadas por los investigadores en la universidad. Esto resulta paradójico en nuestro caso, ya que la mayoría de revistas analizadas corresponden a Asociaciones en las que se encuentran integrados los orientadores. De ahí que se debería potenciar la participación de estos profesionales en la difusión de las experiencias que llevan a cabo en sus centros educativos.

f) Conocer la temáticas más relevantes objeto de investigación en nuestra área, nos permite conocer no sólo el presente, sino también poder tomar decisiones respecto al futuro. Así encontramos que las temáticas que más incidencia tienen son: el Asesoramiento Vocacional y Orientación Vocacional, Programas/Sistemas de Intervención, Asesoramiento/Orientación de la conducta Vocacional y Asesor, equipos y servicios de orientación vocacional. Consideramos que este estos son los campos más relevantes de la Psicología Vocacional, además podemos afirmar que son una constante a lo largo de todos los años estudiados.

Otro aspecto que nos llama la atención es el hecho que campos como la Madurez Vocacional, Información Vocacional y Toma de Decisiones, hayan sido prácticamente olvidados por los investigadores. Y, son precisamente estos campos en los que se debería incidir más en futuras investigaciones.

g) Es significativo que la mayoría de los artículos se adscriben al campo de los Orientadores; así mismo el otro ámbito de mayor incidencia son los estudiantes de Secundaria (esto resulta lógico respecto a cómo está configurada la Orientación en nuestro sistema educativo); si bien, últimamente aparece también un número considerable de artículos relacionados con la Orientación Vocacional en la Universidad. 
h) Es de destacar el carácter eminentemente teórico y empírico de los artículos publicados, siendo mínima la aportación de trabajos prácticos. Entre los trabajos de corte empírico, los tipos de diseños utilizados corresponden a metodologías experimentales y cuasiexperimentales, y metodología de encuestas.

i) Un aspecto que queremos resaltar es el estudio de la indización. Hecho que nos permite trabajar eficazmente con las bases de datos. Al respecto hemos elaborado e incluido en nuestra Base de Datos un glosario de Palabras Clave sobre Orientación Vocacional español, francés e inglés. Las palabras clave más utilizadas hacen referencia a la Orientación Vocacional, Toma de Decisiones y a la Educación Secundaria.

j) De entre los instrumentos que los investigadores han utilizado en sus trabajos, se observa como las Pruebas estandarizadas y los instrumentos de elaboración propia son los más utilizados, centrándose la mayoría de ellos en aspectos relacionados con el desarrollo vocacional de los alumnos. También encontramos una serie de programas dirigidos en su mayoría a la elección vocacional y profesional de los estudiantes. En menor medida, aparecen varias herramientas TIC centradas en la formación de los orientadores.

k) El estudio de las Referencias Bibliográficas es una aportación importante para el conocimiento del Área de Orientación Vocacional. La ficha cientimétrica que hemos utilizado (Flores, 2007) nos ha permitido obtener información de los distintos campos que la integran, así como su interrelación, mostrándose de este modo como una base de datos que nos permite cruzar distintos campos. Los datos más representativos de este apartado son:

- Respecto a los autores más referenciados, estos corresponden a dos grandes grupos. El primero, hace referencia a los autores de otros países, cuya línea de investigación se centra más en la Psicología Vocacional. El segundo, referente al de los autores españoles, se encuentran dentro del ámbito de la Psicopedagogía.

- El idioma utilizado en los documentos de referencia bibliográfica es principalmente el inglés, si bien hay que señalar que encontramos diferencias según el tipo de documento referenciado. Así, en las Revistas el idioma predominante es el inglés, mientras que en los libros el que domina es el español. 
- En cuanto a la "Actualidad" de la bibliografía utilizada, podemos afirmar que, en general, es bastante actual, ya que los autores suelen utilizar documentos de la última década respecto del año de publicación del artículo; y más concretamente los relativos a los últimos cinco años.

- Finalmente, los documentos que sirven de base referencial a los autores se centran principalmente en las Revistas y los Libros.

A tenor de lo expuesto anteriormente, se pone de manifiesto la poca atención que se le ha prestado a la Orientación Vocacional por parte de la Comunidad Científica. Este hecho contrasta con el importante papel que se le ha adjudicado en nuestro país a la Orientación en las distintas etapas de nuestro Sistema Educativo, tanto en los niveles no universitarios (LOE), como muy recientemente en el nivel universitario (Estatuto del Estudiante Universitario), así como a través de las orientaciones que la Comunidad Europea da a través de los distintos informes Trend I (1999), Trend II (2001), Trend III (2003), Trend IV (2005) y Trend V (2007).

En definitiva, creemos que estudios como el que presentamos no sólo dan a conocer dónde estamos sino también qué líneas futuras de trabajo son importantes llevar a cabo para poder dar respuestas eficaces a los retos que a los Orientadores les plantea la sociedad del Siglo XXI. Ya que de este modo, seremos capaces de establecer una comunicación directa entre los investigadores y los profesionales de la Orientación Vocacional, de modo que la ciencia llegue a todos.

\section{Referencias}

Adame, M. T. (2000). Orientación y Psicología Vocacional en España: Revisión y Estado de la cuestión (1970-1999). Tesis doctoral no publicada, Universitat de les Illes Balears, Islas Baleares, España.

Álvárez, M. (1995). Orientación Profesional. [Professional Guidnace]. Barcelona: CEDECS

BOE (1990). Ley Orgánica 1/1990, de 3 de octubre, de Ordenación General del Sistema Educativo.

BOE (2006). Ley Orgánica 2/2006, de 3 de mayo, de Educación. 
Borgen, F. (1991). Megatrends and milestones in vocational behavior: a 20- year counseling psychology perspective. Journal of Vocational Behavior, 39, 263-290

Castaño, C. (1979). 25 años de investigaciones españolas sobre Orientación Vocacional. Revisión Bibliográfica sobre Orientación Vocacional (1952-1978). Encuentros de Psicología Escolar, 1.

Castaño, C. (1983). Psicología y Orientación Vocacional. Madrid: Morava.

Sengupta, I. N. (1992). Bibliometrics, informetrics, scientometrics and librametrics; an overview. Libri, 42 (2), 75-98.

Fernández, A. y Bueno, A. (2002). Multivariate evaluation of Spanish educational research journals. Scientometrics, 55 (1), 87-102.

Flores, R. (2007). La Orientación en España. Una aproximación a través de la Revista Española de Orientación y Psicopedagogía (1990-2006). Trabajo de Investigación no publicado, Universitat Jaume I, Castellón, España.

Flores, R. (2010). Psicología y Orientación Vocacional. Estudio Cientimétrico. Un reto en la Formación del Orientador Europeo del siglo XXI. Tesis Doctoral no publicada, Universitat Jaume I, Castellón, España.

Flores, R., Gil, J.M., Caballer, A. y Martínez, M.A. (2012). The Vocational Guidance Research Database: a Scientometrci Approach. Electronic Journal of Research in Educational Psychology, 10 (1), 397-422.

Nilsson, J E., Flores, L.Y., Berkel, LV., Schale, CL., Linnemeyer, RM. \& Summer, I. (2007). International career articles: A content analysis of four journals across 34 years. Journal of Vocational Behavior, 70, 602-613. http://dx.doi.org/10.1016/j.jvb.2007.01.003

Pascual, J. (1981). Autores más productivos en la psicología actual. En H. Carpintero y J. M. Peiró. Psicología contemporánea. Teoría y métodos cuantitativos para el estudio de su literatura científica. Valencia: Alfaplús.

Reig, F. (1989). Psicología Escolar, Psicología Vocacional y Orientación Escolar a través de las Revistas Bordón, Revista Española de Pedagogía y Revista de Psicología General y Aplicada. (1970-1985). Tesis de Licenciatura no publicada, Universitat de València, Valencia, España.

Rivas, F. (2003). Asesoramiento Vocacional. Teoría, práctica e instrumentación. Barcelona: Ariel.

Terrada, M.L. (1983). La documentación médica como disciplina. Valencia: Centro de Documentación e Información Biomédica de la Universidad de Valencia.

Trends I (1999). Trends in Learning Structures in Higher Education. Recuperado el 22 enero 2009 de http://www.crue.org/export/sites/Crue/procbolonia/documentos/doceua/Trends_I.pdf 
Trends II (2001). Towards the European Higher Education Area - Survey of Main Reforms from Bologna to Prague. Recuperado el 22 enero 2009 de http://www.crue.org/export/sites/Crue/procbolonia/documentos/doceua/Trends_II.pdf

Trends III (2003). Progress towards the European Higher Education Area. Recuperado el 22 enero 2009 de http://www.crue.org/export/sites/Crue/procbolonia/documentos/doceua/Trends_III.pdf

Trends IV (2005). European Universities Implementing Bologna. Recuperado el 22 enero 2009 de http://www.crue.org/export/sites/Crue/procbolonia/documentos/doceua/Trends_IV.pdf

Trends V (2007). Universities Shaping the European Higher Education Area. Recuperado el 22 enero 2009 http://www.crue.org/export/sites/Crue/procbolonia/documentos/doceua/EUA_Trends_V_for_web.pdf

Vickery, B. C. (1948). Bradford's law of scattering. Journal of Documentation, 4 (1), 198203.

Vinkler, P (1991). Possible causes of differences in information impact of journals from different subfields. Scientometrics, 20 (2), 145-161. 